\title{
Planetary Gear Trains - the Main Problems Gathered in a Book
}

\section{Dimitar Karaivanov*}

Department of Applied Mechanics, University of Chemical Technology and Metallurgy (UCTM), Sofia, Bulgaria

*Corresponding Author: Dimitar Karaivanov, Department of Applied Mechanics, University of Chemical Technology and Metallurgy

(UCTM), Sofia, Bulgaria.

Received: March 28, 2019; Published: June 06, 2019

DOI: 10.31080/ASAG.2019.03.0517

\begin{abstract}
Planetary gear trains are used in many areas of the technic including in the agriculture and food-processing machinery. The big number of types and great diversity of design solution make the choice of the most appropriate arrangement very difficult. A big number of articles, dissertations, books on the planetary gear trains exist. In more cases they carried out a separate problem or particular arrangement of planetary gear train. This review presents a book in which the main problems of almost all of used in practice cylindrical planetary gear trains are discussed. The book is addressed to engineers (design and industrial application) as well as to students - master and $\mathrm{PhD}$ degrees. The book is result of both scientific and practical experience of the authors. It was written under the motto "From practice to practice".
\end{abstract}

Keywords: Planetary Gear Train; Efficiency; Kinematic Analysis; Power Analysis; Speed Ratio; Transmission

\section{Abbreviations}

PGT: Planetary Gear Train.

\section{Introduction}

Planetary gear trains (PGTs) have some advantages which make them appropriate for using in agricultural machines and equipment as well as in the food-processing industry:

- $\quad$ Torque split leads to smaller mesh load and from here to smaller overall dimensions, e.g. to:

- Smaller weight;

- Lower peripheral velocities of gears, e.g.

- Lower dynamic loading;

- Lower noise and vibrations;

- $\quad$ Lower relative sliding (high efficiency);

- Easily fabrication of units, mainly of gears, and from here to:

- $\quad$ High accuracy (e.g. lower noise and vibrations; high efficiency; high durability and reliability);

- $\quad$ Easy thermal treatment;

- Coaxiality of the input and output shafts is very appropriate for some transmissions.
- $\quad$ Planetary type of movement (revolution around the central axis and spin) of planet gears is very useful in technological machines, stirrers, etc.

Except mentioned advantages some disadvantages of PGTs are in force:

- More complex theory (in comparison with non-planetary gear trains):

- Internal meshing;

- Problems with power pat identification in complex compound multicarrier PGTs;

- Problems with kinematic and efficiency analysis;

- Necessity of high quality fabrication (in case of low accuracy many of advantages are not actual);

- Possible problems with uniformity of load sharing between planets (the torque split advantage may be rejected);

- The compactness (one of advantages) means smaller cooling surface, i.e., overheating hazard.

All these advantages and disadvantages must be known for adequate application of PGTs including in agricultural machinery. 
Additional difficult is a big number of types of PGTs with diametric opposing properties.

PGTs problematic is investigated by numerous articles, dissertation, books. Some of them accented on particular problems (load sharing, efficiency, dynamics, etc.). Others treat particular type or arrangement. Third are on very high "scientific" level, not useful for engineers from industry.

All of the above arouse a necessity of a book that presents the greatest possible number of PGTs, as well as to thoroughly examine the most interesting problems of the most common used ones.

Prof. Kiril Arnaudov from Bulgarian Academy of Sciences (Institute of Mechanics) and Prof. Dimitar Karaivanov from University of Chemical Technology and Metallurgy (Department of Applied Mechanics) have endeavored to respond to this necessity. The book Planetary Gear Trains has been published in Bulgarian [1] in 2017 and has been translated in English and published [2] in 2019.

\section{Book structure}

The text of both edition (Bulgarian and English) is arranged in five parts as follows:

Part I - "General", consist of two chapters: "Introduction" and "Nature, Purpose, and Types of Planetary Gear Trains".

Part II - "AI -Planetary Gear Train". This part in eighteen chapters presents the most common used planetary gear train (Figure 1) - from "Arrangement and Possible Ways of Work" and "Specific Conditions" (Assembly, Coaxiality, and Adjacent conditions) through "Load Distribution between the Planets" and "Efficiency" to "Optimization" and "Examples for Application". In accepted designation letter A means external meshing (between sun gear 1 and planets 2), letter I - internal meshing (between planets 2 and ring gear 3), and a line above - one-rim planet [3].

Part III - "Other Types of Simple Cylindrical Planetary Gear Trains". Not all possible, but most of the practically used types of simple PGTs are considered. All of these seven chapters present kinematic scheme (arrangement), speed ratio and efficiency determination, special conditions (assembly condition is more interesting), some special features (about internal meshing, mainly), advantages, disadvantages, and application of PGTs in question. The aim of authors was to include all the information about one type of PGT in corresponding chapter so that a reader can receive complete clarity on them and creatively understand them.

Part IV - "Compound Planetary Gear Trains". From the vast multitude of compound PGTs here the three possible cases of two- carrier PGTs (Figure 2) as well as a few useful structural schemes of three-carrier PGTs are considered. A special attention is paid to so-called reduced PGTs (Wolfrom and Ravigneaux gear trains) - two-carrier PGTs in which both carriers of the component PGTs are merged (reduced) into a single carrier. The last chapter of this part consists of fifteen examples of application of two- three- and four-carrier PGTs with comments of their features and advices for application.

Part V - "Supplements" briefly presents some interesting cases of bevel, worm, and crossed-helical simple PGTs, other special PGTs (bi-planetary and precesional) and last but not least asymmetric teeth application in PGTs.
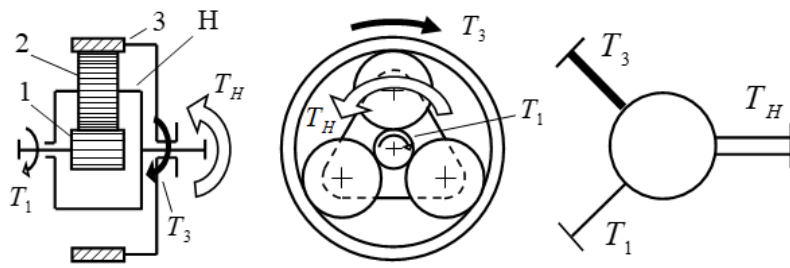

Figure 1: AI -planetary gear train, its structural symbol, and torques at its external shafts (1, 3 and $\mathrm{H}$ ): 1 - sun gear; 2 - planet; 3 - ring gear; $\mathrm{H}$ - carrier.

More interesting points in the book

The presented book has discussed theory, calculations and design, load capacity and durability, manufacturing and quality control, applications of different types of elementary (simple, singlecarrier) and complex compound (multi-carrier) PGTs. But there are a few special points, result of the authors' investigations and publications over the years.

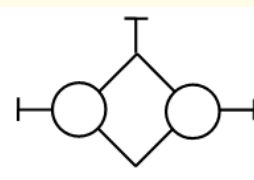

a)

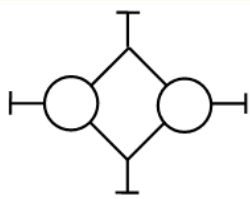

b)

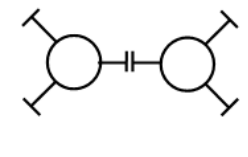

c)
Figure 2: Possible cases of connecting component PGTs in compound two-carrier PGT: a) with two compound and three external shafts; b) with two compound and four external shafts; c) with one compound and four external shafts. 
Torque method for investigation of planetary gear trains

The idea of using the torques of the outer shafts of a PGT to determine its gear ratio is not a new one. The authors developed it [4-6] for power flows investigation and efficiency determination. Except in the book in question one can find a detailed description of the method in [7]. The torque method has some advantages:

- $\quad$ Simple and easy for use;

- Gives a possibility for results verification at every stage of calculations;

- $\quad$ Allows the speed ratio determination as well as the efficiency determination;

- Allows easy determination of direction and value of internal power flows in closed-power-loop compound PGTs and on this base determination of internal division or internal circulation of power presence.

All the kinematic and efficiency analysis in the chapters of Part IV are made by torque method.

\section{Load distribution between the planets}

Twenty three pages to this interesting and very important problem are spared. Causes for uneven load distribution, influencing factors, and possibilities of its theoretical and experimental determination are considered. An original test rig (developed by K. Aknaudov) with very width possibilities for influence factors investigation is presented. Ways of teeth loading experimental determination are discussed. Ways of problem solving and advices for unevenness considering in load capacity calculations (according to ISO 6336) are given. Special attention is pointed on the various equalizing devices characteristics.

\section{Efficiency determination}

In a chapter the type of losses and basic efficiency (in work with fixed carrier) of AI -PGT are considered. Various formulae for theoretical determination of meshing loss are given. In another chapter theoretical and experimental ways for efficiency determination are presented. In theoretical determinations it is pointed on the scattering of friction coefficient and scattering of the efficiency as a result. Several methods for experimental determination of the efficiency of PGTs are discussed. Because the very high efficiency of $\overline{\mathrm{AI}}$-PGT (97-98\%) the method with input and output torques measuring is not appropriate. Two rigs for efficiency determination by thermal method (developed by K. Arnaudov) and for thermal and static methods (developed by D. Karaivanov) are presented.

\section{Conclusion}

As is mentioned above presented book is written from practice to practice. It is very richly illustrated (authors consider that an illustration gives more information than a page text) in order to be understandable and accessible to the widest possible range of readers - scientists, engineers, students. Author of this review considers the book will be interesting for engineers and students from the field of agricultural machinery because PGTs are used there. Figure 3 presents $\overline{\mathrm{AI}}$-PGT as inter-wheel reducer of tractor. The same type of PGT is a part of steering mechanism of continuous track vehicles (Figure 4). And, of course, classical inter-wheel differential, in the rare axle of a tractor, for example (Figure 5) is planetary too. PGTs can be found in several special devices of fertilizer [8] and other [9] agricultural machines.

\section{Acknowledgements}

The author would like to express his deepest gratitude to Both publishers of Bulgarian and English editions of the book;

- $\quad$ Eng. Georgi Stoimenov and Podemcrane AD, who sponsored the publication of the Bulgarian edition as well as its translation into English;

- $\quad$ Prof. Dr. Marin Guenov and Eng. Vladimir Tzanov for advising about the terminology used in their scientific and engineering field;

- The gear experts and colleagues from Balkan Association of Power Transmissions, with whom it was a pleasure to exchange experience and ideas at numerous scientific conferences

\section{Conflict of Interest}

No financial interest and conflict of interest exist.

\section{Bibliography}

1. Arnaudov K and D Karaivanov. "Planetary gear trains". Sofia: Bulgarian Academy of Sciences Publ. "Prof. Marin Drinov", (2017): 368

2. Arnaudov K and D Karaivanov. "Planetary gear trains". Boca Raton FL: CRC Press, (2019): 358.

3. Tkachenko VA. "Design of multi-planet planetary gear trains". Kharkov: Kharkov National University Publ., (1961).

4. Arnaudov K. "Engineering analysis of compound (two-carrier) planetary gear trains". Symposium "Heavy Machinery". Varna [Bulgaria], (1984). 
5. Karaivanov D. "Theoretical and experimental studies of influence of the structure of coupled two-carrier planetary gear trains on its basic parameters". Dissertation. Sofia: University of Chemical Technology and Metallurgy, (2000).

6. Arnaudov K and D Karaivanov. "The Torque Method Used for Studying Coupled Two-Carrier Planetary Gear Trains". Transactions of FAMENA 37.1 (2013): 49-61.

7. Arnaudov K and D Karaivanov. "Torque method for analysis of compound planetary gear trains". Beau Basin [Mauritius]: LAP LAMBERT Academic Publishing, (2017): 92.

8. Eskhozhin D., et al. "Design and study of a dispenser for the introduction of the main batch of mineral fertilizers". Mechanika 24.3 (2018): 343-351.

9. Turymbetova G. "Justification of the type of rice-harvesting combines for rice farms of the Kyzylorda region". Dissertation. Almaty [Kazakhstan]: KazNAU, (2018).

Volume 3 Issue 7 July 2019

(C) All rights are reserved by Dimitar Karaivanov. 